\title{
Desirable Dog-Rabies Control Methods in an Urban setting in Africa - a Mathematical Model
}

\author{
Edwiga Kishinda Renald ${ }^{\mathrm{a}, \mathrm{b}}$, Dmitry Kuznetsov ${ }^{\mathrm{a}}$, Katharina Kreppel ${ }^{\mathrm{c}}$ \\ ${ }^{a}$ School of CoCSE, Department of Applied Mathematics and Computational Sciences, Nelson Mandela African \\ Institution of Science and Technology, P.O.Box 447, Arusha, Tanzania \\ ${ }^{b}$ Mathematics and Statitstics Department, Mwenge Catholic University, P.O.Box 1226, Moshi, Tanzania \\ 'School of LISBE, Department of Global Health and Biomedical Sciences, Nelson Mandela African Institution \\ of Science and Technology, P.O.Box 447, Arusha, Tanzania
}

Received: 25 July 2019; Accepted: 11 August 2019; Published: 08 February 2020

\begin{abstract}
Rabies is a fatal, zoonotic, viral disease that causes an acute inflammation of the brain in humans and other mammals. It is transmitted through contact with bodily fluids of infected mammals, usually via bites or scratches. In this paper, we formulate a deterministic model which measures the effects of different rabies control methods (mass-culling and vaccination of dogs) for urban areas near wildlife, using the Arusha region in Tanzania as an example. Values for various parameters were deduced from five years' worth of survey data on Arusha's dog population. Data included vaccination coverage, dog bites and rabies deaths recorded by a local non-governmental organization and the Ministry of Agriculture, Livestock Development and Fisheries of the United Republic of Tanzania. The basic reproduction number $R_{0}$ and effective reproduction number $R_{e}$ were computed and found to be 1.9 and 1.2 respectively. These imply that the disease is endemic in Arusha. The numerical simulation of the reproduction number shows that vaccination is the most appropriate control method for rabies transmission in urban areas near wildlife reservoirs. The disease free equilibrium $\varepsilon_{0}$ is also computed. If the effective reproduction number $R_{e}$ is computed and found to be less than 1 , it implies that it is globally asymptotically stable in the feasible region $\Phi$. If $R_{e}>1$ it is implied that there is one equilibrium point which is endemic and it is locally asymptotically stable.
\end{abstract}

Index Terms: Rabies, Vaccination, Culling for Dog Control, SEIV-Model, Reproduction Number, Arusha.

(C) 2020 Published by MECS Publisher. Selection and/or peer review under responsibility of the Research Association of Modern Education and Computer Science

\footnotetext{
* Corresponding author.
}

E-mail address: 


\section{Introduction}

Mathematical modeling has historically been of great importance in epidemiology and is a useful tool for providing a better insight into the dynamics of epidemic diseases such as rabies.

Rabies is a fatal, zoonotic, viral disease that causes an acute inflammation of the brain in humans and other mammals. It is transmitted by the saliva of infected animals via bites or scratches, with dogs being the primary source of transmission to humans [23].Rabies occurs in more than 150 countries and territories around the world and is most prevalent in developing countries in Africa and Asia [8].

Globally, it claims an estimated 60,000 human lives annually [15], the highest number of deaths caused by any zoonotic disease [11,16]. Dog transmitted rabies is estimated to cause 24,000 human deaths per year in Africa and [26] up to $60 \%$ of dog bite victims are children less than 15 years of age. Unfortunately, the majority of dog bites go unreported to parents and any resulting rabies cases are not reported to health authorities [2]. In Tanzania, it claims the lives of around 1500 people yearly [19].

The two main ways to control rabies transmission are mass-dog vaccination and culling, whereby the culling method is perceived to be easier and cheaper than vaccination, especially in the presence of free-roaming and poorly socialized animals in areas where veterinary personnel has relatively little experience or confidence in handling dogs [18].

Despite these control efforts, rabies remains a problem with 99\% of all human deaths from rabies occurring in the developing world [14].

However, according to Mbwa wa Africa, an animal welfare organization in Arusha conducting research, every killed dog is replaced within 6 months by a new, young dog [22]. As dogs are territorial and defend their resting and feeding grounds in packs, killed members of a pack affect its ability to hold a territory, leading to more fighting and mixing of the overall dog population. Killing a neutered, vaccinated dog, therefore often leads to its replacement by an unvaccinated, unneutered dog, potentially increasing the risk for rabies outbreaks [22].

In order to reduce the risk of transmission and keep rabies control costs as low as possible, information on the efficacy of culling and mass dog vaccination programs is required. We used a mathematical model to establish the impact of culling and vaccination in Arusha respectively.

A similar model, but in a very different environment, was formulated to describe the dynamics of rabies transmission among dogs, livestock and humans within and around Addis Ababa, Ethiopia [8]. The model predicted an increase in rabies transmission with a maximum prevalence in 2024 and 2026 for both humans and livestock respectively and a combination of interventions was suggested. In another study, [12] a susceptibleexposed-infectious-vaccinated (SEIV) model for dog-human transmission of rabies considering domestic and stray dogs was proposed and showed that rabies in Guangdong province in China would decrease gradually before increasing again, indicating that in this case culling for disease control is futile. Differences in the dog populations, especially with regards to roaming patterns and contacts with wildlife areas require different modelling approaches to fit the conditions.

The specificity of our research considers three subgroups of dogs; domestic dogs with clear owners, stray dogs roaming the streets and Maasai dogs travelling alongside livestock and herdsmen. In this study, dog mass vaccination has been compared to stray dog culling in terms of its effects on rabies transmission risk.

\section{Materials and Methods}

\subsection{Model Formulation}

We developed a basic transmission risk model tailored to areas with similar settings as Arusha, to measure the effect of culling and vaccination. The formulated model has three dog subgroups, which are domestic dogs, stray dogs and Maasai dogs. Each population is categorized into Susceptible, Exposed, Infectious and Vaccinated individuals and a SEIV model was formulated. 
The susceptible class consists of currently disease free individuals. The exposed class contains individuals who have been contracted the virus but do not show symptoms of the disease. The infectious class consists of individuals who were exposed to the disease, developed clinical symptoms of rabies and will die. Finally, the vaccinated class consists of individuals formally susceptible or exposed to the disease but now vaccinated. The formulated model is a system of differential equations, which has been derived from the compartmental diagram in Figure 1.

The model is developed based on the following assumptions; the susceptible populations are recruited via birth rate $\alpha$. Any kind of dog which is exposed to bodily fluid from another dog is exposed. Dogs in each group have equal probability of dying a natural death. Populations are considered homogeneous with regard to each dog's probability of being infected. Once a dog reaches the infectious stage, death is $100 \%$ certain. All parameters of the model are positive and they are introduced in table 1.

\section{TABLE 1. PARAMETER DESCRIPTION}

\begin{tabular}{cl}
\hline Parameter & Description \\
\hline$\alpha_{d}, \alpha_{s}, \alpha_{m}$ & Annual births of domestic dog, stray dog and Maasai dog populations respectively. \\
$\delta_{d}, \delta_{s}, \delta_{m}$ & Death rate due to rabies for domestic dog, stray dog and Maasai dog populations respectively. \\
$\omega_{d}, \omega_{s}, \omega_{m}$ & The loss rate of vaccination immunity for domestic dog, stray dog and Maasai dog populations respectively. \\
$\mu_{d}, \mu_{s}, \mu_{m}$ & Natural death rate of domestic dog, stray dog and Maasai dog populations respectively. \\
$\beta_{d}, \beta_{s}, \beta_{m}$ & $\begin{array}{l}\text { Rate at which infectious stray dogs infect susceptible domestic dog, stray dog and Maasai dog populations } \\
\text { respectively. }\end{array}$ \\
$\rho_{d}, \rho_{s}, \rho_{m}$ & The incubation period in domestic dog, stray dog and Maasai dog populations respectively. \\
$\sigma_{d}, \sigma_{s}, \sigma_{m}$ & Vaccination rate of susceptible domestic dog, stray dog and Maasai dog populations respectively. \\
$\Psi_{m d}, \Psi_{s d}, \Psi_{d s}, \Psi_{m s}$ & $\begin{array}{l}\text { Number of dogs migrated from Maasai to domestic, stray to domestic, domestic to stray and Maasai to stray } \\
\text { dogs' populations respectively. } \\
\mu_{c}\end{array}$ \\
Average culling rate of stray dogs.
\end{tabular}

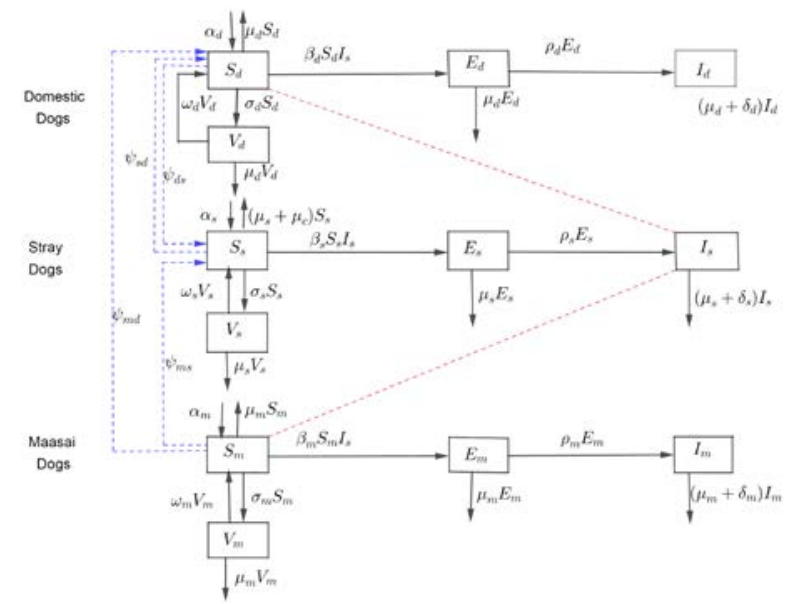

Fig.1. Flow diagram for rabies transmission among dog subgroups.

\subsection{Model Compartment and Dynamics}

From the above assumptions, definition of variables and parameters, the model flow diagram depicts the 
dynamics of rabies transmission among domestic dogs, stray dogs and Maasai dogs as shown in Figure 1.Parameters $\alpha \mathrm{i}$ where, $\mathrm{i}=\mathrm{d}, \mathrm{s}, \mathrm{m}$ represent annual births of domestic dog, stray dog and Maasai dog populations respectively. The parameters $\rho \mathrm{i}$ where, $\mathrm{i}=\mathrm{d}, \mathrm{s}, \mathrm{m}$ represent the latency rates of domestic dogs, stray dogs and Maasai dogs so that $1 / \rho \mathrm{i}$ where, $\mathrm{i}=\mathrm{d}, \mathrm{s}, \mathrm{m}$ are the corresponding incubation periods.

\subsection{Model Equations}

From the compartmental diagram we formulate a set of twelve differential equations as shown below:

$$
\left\{\begin{array}{l}
\frac{d S_{d}}{d t}=\alpha_{d}+\omega_{d} V_{d}+\Psi_{s d}+\Psi_{m d}-\Psi_{d s}-\left(\mu_{d}+\sigma_{d}+\beta_{d} I_{s}\right) S_{d} \\
\frac{d E_{d}}{d t}=\beta_{d} S_{d} I_{s}-\left(\mu_{d}+\rho_{d}\right) E_{d} \\
\frac{d I_{d}}{d t}=\rho_{d} E_{d}-\left(\mu_{d}+\delta_{d}\right) I_{d} \\
\frac{d V_{d}}{d t}=\sigma_{d} S_{d}-\left(\omega_{d}+\mu_{d}\right) V_{d} \\
\frac{d S_{s}}{d t}=\alpha_{s}+\omega_{s} V_{s}+\Psi_{d s}+\Psi_{m s}-\Psi_{s d}-\left(\sigma_{s}+\mu_{s}+\mu_{c}+\beta_{s} I_{s}\right) S_{s} \\
\frac{d E_{s}}{d t}=\beta_{s} S_{s} I_{s}-\left(\mu_{s}+\rho_{s}\right) E_{s} \\
\frac{d I_{s}}{d t}=\rho_{s} E_{s}-\left(\mu_{s}+\delta_{s}\right) I_{s} \\
\frac{d V_{s}}{d t}=\sigma_{s} S_{s}-\left(\omega_{s}+\mu_{s}\right) V_{s} \\
\frac{d S_{m}}{d t}=\alpha_{m}+\omega_{m} V_{m}-\Psi_{m s}-\Psi_{m d}-\left(\mu_{m}+\sigma_{m}+\beta_{m} I_{s}\right) S_{m} \\
\frac{d E_{m}}{d t}=\beta_{m} S_{m} I_{s}-\left(\mu_{m}+\rho_{m}\right) E_{m} \\
\frac{d I_{m}}{d t}=\rho_{m} E_{m}-\left(\mu_{m}+\delta_{m}\right) I_{m} \\
\frac{d V_{m}}{d t}=\sigma_{m} S_{m}-\left(\omega_{m}+\mu_{m}\right) V_{m}
\end{array}\right.
$$

with,

$$
\begin{aligned}
N_{d}(t) & =S_{d}(t)+E_{d}(t)+I_{d}(t)+V_{d}(t) \\
N_{s}(t) & =S_{s}(t)+E_{s}(t)+I_{s}(t)+V_{s}(t) \\
N_{m}(t) & =S_{m}(t)+E_{m}(t)+I_{m}(t)+V_{m}(t)
\end{aligned}
$$

Where $N_{i}, i=d, s, m$ is the total of domestic dogs, stray dogs and Maasai dogs' population at time $t$.

\subsection{Invariant Region}

The model represented by the system 1 of differential equations which deals with domestic dogs, stray dogs 
and Maasai dogs, will be analyzed in the feasible region $\Phi$ and all state variables and parameters are assumed to be positive for all $t \geq 0$. The invariant region will be obtained through Theorem 1 .

\section{Theorem 1}

All solutions of the system 1 are contained in the region $\Phi \in \mathbb{R}^{12}$ and $\Phi=\Phi_{\mathrm{d}} \cup \Phi_{\mathrm{s}} \cup \Phi_{\mathrm{m}}$

\section{Proof}

The model of the system 1 was grouped into domestic dogs $N_{d}$, stray dogs $N_{s}$ and Maasai $\operatorname{dogs} N_{m}$, such that

$$
\begin{aligned}
& \Phi_{d}=\left\{\left(S_{d}, E_{d}, I_{d}, V_{d}\right) \in \square_{+}^{4}: 0 \leq N_{d} \leq \frac{\alpha_{d}}{\mu_{d}}\right\} \\
& \Phi_{s}=\left\{\left(S_{s}, E_{s}, I_{s}, V_{s}\right) \in \square_{+}^{4}: 0 \leq N_{s} \leq \frac{\alpha_{s}}{\mu_{s}}\right\} \\
& \Phi_{m}=\left\{\left(S_{m}, E_{m}, I_{m}, V_{m}\right) \in \square_{+}^{4}: 0 \leq N_{m} \leq \frac{\alpha_{m}}{\mu_{m}}\right\}
\end{aligned}
$$

And $\Phi$ is the positive invariant region for system 1.

Thus,

$$
\Phi=\Phi_{d} \cup \Phi_{s} \cup \Phi_{m} \in \square_{+}^{4} \times \square_{+}^{4} \times \square_{+}^{4}
$$

From that, it is sufficient to consider model system 1 in the region $\Phi$, and it can be shown to be positively invariant. The model can be considered as epidemiologically and mathematically well-posed.

\section{Model Analysis}

\subsection{Disease Free Equilibrium Points (DFE)}

To find the disease free equilibrium points we set the right hand side of equations of system 1 equal to zero. In the absence of attack or in the absence of rabies, $E_{d}=I_{d}=V_{d}=E_{s}=I_{s}=E_{m}=I_{m}=V_{m}=0$. Then the disease free equilibrium (DFE) $\varepsilon_{0}$ will be $\varepsilon_{0}=\left(S_{d}^{0}, 0,0,0, S_{s}^{0}, 0,0, V_{s}^{0}, S_{m}^{0}, 0,0,0\right)$

where

$$
\begin{aligned}
& S_{d}^{0}=\frac{\alpha_{d}-\Psi_{d s}+\Psi_{m d}+\Psi_{s d}}{\mu_{d}+\sigma_{d}}, S_{s}^{0}=\frac{\left(\mu_{s}+\omega_{s}\right)\left(\Psi_{d s}-\Psi_{s d}+\Psi_{m s}+\alpha_{s}\right)}{\mu_{c}\left(\mu_{s}+\omega_{s}\right)+\mu_{s}\left(\mu_{s}+\sigma_{s}+\omega_{s}\right)}, \\
& V_{s}^{0}=\frac{\alpha_{s}\left(\Psi_{d s}+\Psi_{m s}+\alpha_{s}-\Psi_{s d}\right)}{\mu_{c}\left(\mu_{s}+\omega_{s}\right)+\mu_{s}\left(\mu_{s}+\sigma_{s}+\omega_{s}\right)}, S_{m}^{0}=\frac{\alpha_{m}-\Psi_{m d}-\Psi_{m s}}{\mu_{m}+\sigma_{m}}
\end{aligned}
$$

The disease free equilibrium points for stray dogs populations that is $V_{S}$ cannot be zero because once susceptible stray dog is vaccinated, it transfer to the vaccinated class. Hence the disease free equilibrium point of the system 1 exists and it is given by 


$$
\begin{aligned}
\varepsilon_{0}= & \left(\frac{\alpha_{d}-\Psi_{d s}+\Psi_{m d}+\Psi_{s d}}{\mu_{d}+\sigma_{d}}, 0,0,0, \frac{\left(\mu_{s}+\omega_{s}\right)\left(\Psi_{d s}-\Psi_{s d}+\Psi_{m s}+\alpha_{s}\right)}{\mu_{c}\left(\mu_{s}+\omega_{s}\right)+\mu_{s}\left(\mu_{s}+\sigma_{s}+\omega_{s}\right)}, 0,0,\right. \\
& \left.\frac{\alpha_{s}\left(\Psi_{d s}+\Psi_{m s}+\alpha_{s}-\Psi_{s d}\right)}{\mu_{c}\left(\mu_{s}+\omega_{s}\right)+\mu_{s}\left(\mu_{s}+\sigma_{s}+\omega_{s}\right)}, \frac{\alpha_{m}-\Psi_{m d}-\Psi_{m s}}{\mu_{m}+\sigma_{m}}, 0,0,0\right)
\end{aligned}
$$

\subsection{The Basic Reproduction Number $R_{0}$}

The basic reproduction number R0 can be defined as the expected number of secondary infections produced by an index case in a completely susceptible population [24]. The basic reproduction number can be used to assess whether a newly infectious disease can invade a population [3]. $\mathrm{R}_{0}<1$ implies that, on average, an infected individual results in less than one newly infected individual during its infectious period, and the infection cannot grow. Conversely, if $\mathrm{R}_{0}>1$, on average, each infected individual creates more than one new infection, and the disease can raid the population. We used a next generation operator method proposed by Van den Driessche and Watmough (2000) [27].

We considered system 1 without vaccination i.e. $\omega=\sigma=0$. In this case we also do not have culling, which means $\mu_{\mathrm{c}}=0$.

Let $f_{i}(x)$ be the rate of appearance of new infection in compartment $i, v_{i}^{-}(x)$ be the rate of transfer of individuals out of compartment $i$ and $v_{i}^{+}(x)$ be the rate of transfer of individuals into compartment $i$ by all other means, and it is assumed that each function is continuously differentiable at least twice in each variable. The disease transmission model of system 1 consists of non-negative initial conditions together with the following system of equations: $\dot{x}=F_{i}(x)=f_{i}(x)-v_{i}(x)$ wherev $_{i}=v_{i}^{-}-v_{i}^{+}$. We now consider expressions in which the infection is in progress. That is $E_{d}, I_{d}, E_{s}, I_{s}, E_{m}, I_{m}$

$$
\begin{aligned}
\frac{d E_{d}}{d t} & =\beta_{d} S_{d} I_{s}-\left(\mu_{d}+\rho_{d}\right) E_{d} \\
\frac{d I_{d}}{d t} & =\rho_{d} E_{d}-\left(\mu_{d}+\delta_{d}\right) I_{d} \\
\frac{d E_{s}}{d t} & =\beta_{s} S_{s} I_{s}-\left(\mu_{s}+\rho_{s}\right) E_{s} \\
\frac{d I_{s}}{d t} & =\rho_{s} E_{s}-\left(\mu_{s}+\delta_{s}\right) I_{s} \\
\frac{d E_{m}}{d t} & =\beta_{m} S_{m} I_{s}-\left(\mu_{m}+\rho_{m}\right) E_{m} \\
\frac{d I_{m}}{d t} & =\rho_{m} E_{m}-\left(\mu_{m}+\delta_{m}\right) I_{m}
\end{aligned}
$$

By rearranging equations of system 1 without vaccination from exposed to infectious classes of dogs' subgroups with a system of equations given by 7 . Let $\mathrm{F}$ be a non-negative $\mathrm{n} \times \mathrm{n}$ matrix and $\mathrm{V}$ be a non-singular $\mathrm{N}$-matrix such that

$$
F=\left[\frac{\partial f_{i}\left(\varepsilon_{0}\right)}{x_{j}}\right] \text { and } V=\left[\frac{\partial v_{i}\left(\varepsilon_{0}\right)}{x_{j}}\right]
$$


with $1 \leq \mathrm{i}, \mathrm{j} \leq \mathrm{n}$. The point $\varepsilon_{0}$ is the disease free equilibrium point in 6 without vaccination where

$$
f_{i}=\left[\begin{array}{c}
\beta_{d} S_{d} I_{s} \\
0 \\
\beta_{s} S_{s} I_{s} \\
0 \\
\beta_{m} S_{m} I_{s} \\
0
\end{array}\right] \text { and } v_{i}=\left[\begin{array}{c}
\left(\mu_{d}+\rho_{d}\right) E_{d} \\
\left(\mu_{d}+\delta_{d}\right) I_{d}-\rho_{d} E_{d} \\
\left(\mu_{s}+\rho_{s}\right) E_{s} \\
\left(\mu_{s}+\delta_{s}\right) I_{s}-\rho_{s} E_{s} \\
\left(\mu_{m}+\rho_{m}\right) E_{m} \\
\left(\mu_{m}+\delta_{m}\right) I_{m}-\rho_{m} E_{m}
\end{array}\right]
$$

We consider classes in which the disease is in progress. Using the linearization technique, we get the Jacobian matrices of $f$ and $v$ at the disease free equilibrium point $\varepsilon_{0}$ as shown below:

$$
F=\left[\begin{array}{cccccc}
0 & 0 & 0 & \frac{\beta_{d}\left(\alpha_{d}-\Psi_{d s}+\Psi_{m d}+\Psi_{s d}\right)}{\mu_{d}} & 0 & 0 \\
0 & 0 & 0 & 0 & 0 & 0 \\
0 & 0 & 0 & \frac{\beta_{s}\left(\alpha_{s}+\Psi_{d s}+\Psi_{m d}-\Psi_{s d}\right)}{\mu_{s}} & 0 & 0 \\
0 & 0 & 0 & 0 & 0 & 0 \\
0 & 0 & 0 & \frac{\beta_{m}\left(\alpha_{m}-\Psi_{m s}-\Psi_{m d}\right)}{\mu_{m}} & 0 & 0 \\
0 & 0 & 0 & 0 & 0 & 0
\end{array}\right], V=\left[\begin{array}{cccccc}
\mu_{d}+\rho_{d} & 0 & 0 & 0 & 0 & 0 \\
-\rho_{d} & \mu_{d}+\delta_{d} & 0 & 0 & 0 & 0 \\
0 & 0 & \mu_{s}+\rho_{s} & 0 & 0 & 0 \\
0 & 0 & -\rho_{s} & \mu_{s}+\delta_{s} & 0 & 0 \\
0 & 0 & 0 & 0 & \mu_{m}+\rho_{m} & 0 \\
0 & 0 & 0 & 0 & -\rho_{s} & \mu_{m}+\delta_{m}
\end{array}\right]
$$

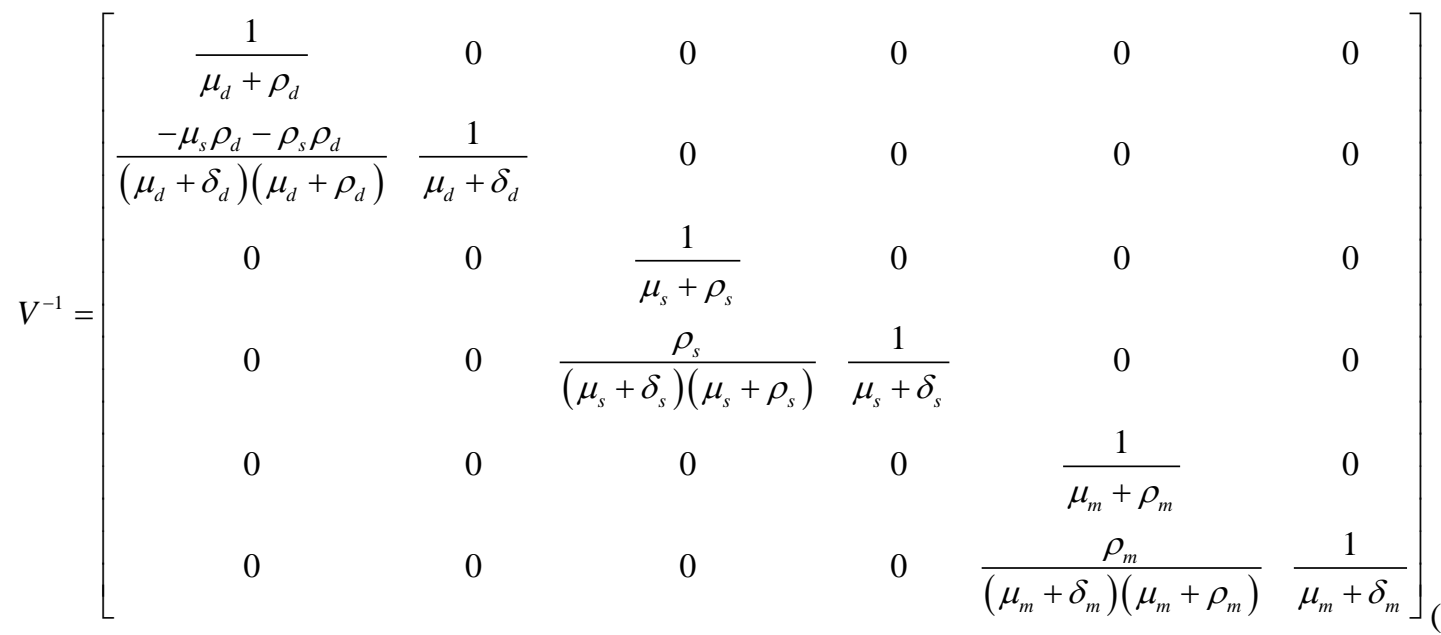

We now multiply $F$ and $V^{-1}$ and then compute the Eigen values of the resulting matrix $F V^{-1}$ and choose the maximum Eigen value as the basic reproduction number $R_{0}$ which is given by

$$
R_{0}=\frac{\beta_{s} \rho_{s}\left(\Psi_{d s}+\Psi_{m s}+\alpha_{s}-\Psi_{s d}\right)}{\mu_{s}\left(\mu_{s}+\delta_{s}\right)\left(\mu_{s}+\rho_{s}\right)}
$$




\subsection{Effective Reproduction Number $R_{e}$}

The effective reproduction number $\mathrm{R}_{\mathrm{e}}$ can be defined as the average number of secondary cases that one index case generates over the course of its infectious period [7]. The prevalence of infection increases or decreases according to whether $\mathrm{R}_{\mathrm{e}}$ is greater than or less than one, respectively [6]. Here we consider the presence of control methods. In our case we have vaccination and culling. In this case $\omega, \mu_{c}$ and $\sigma$ will not take on zero values. So we include them and follow the same procedures usedin computing $R_{0}$ and this will result in the spectral radius (dominant Eigen value) $\mathrm{R}_{\mathrm{e}}=\rho \mathrm{FV}^{-1}$ of $\mathrm{FV}^{-1}$ given by

$$
R_{e}=\frac{\beta_{s} \rho_{s}\left(\mu_{s}+\omega_{s}\right)\left(\Psi_{d s}+\Psi_{m s}+\alpha_{s}-\Psi_{s d}\right)}{\left(\mu_{s}+\delta_{s}\right)\left(\mu_{s}+\rho_{s}\right)\left(\mu_{c} \omega_{s}+\mu_{s} \mu_{c}+\mu_{s} \sigma_{s}+\mu_{s} \omega_{s}+\mu_{s}^{2}\right)}
$$

Numerical computations of $\mathrm{R}_{0}$ and $\mathrm{R}_{\mathrm{e}}$ were done using the data collected from Mbwa wa Africa and the Ministry of Agriculture, Livestock Development and Fisheries of The United Republic of Tanzania.

\section{TABLe 2. VALUes OF PARAMETERS USED AT DFE}

\begin{tabular}{|c|c|c|c|}
\hline & Parameter & Value $\left(\right.$ year $\left.^{-1}\right)$ & Source \\
\hline$\alpha_{s}$ & The annual births of stray dogs & $2.5 \times 10^{2}$ & {$[22]$} \\
\hline$\delta_{s}$ & Death rate due rabies for stray dogs & 0.22 & [4] \\
\hline$\omega_{s}$ & Loss rate of vaccination immunity for stray dogs & 0.1 & Assumption \\
\hline$\mu_{s}$ & Natural death rate of stray dogs & 0.32 & {$[20]$} \\
\hline$\beta_{s}$ & Rate of infection of stray dogs & $1.7864 \times 10^{-4}$ & Data \\
\hline$\rho_{s}$ & The incubation period of stray dog & 0.83778234 & {$[17]$} \\
\hline$\sigma_{s}$ & Vaccination rate of the susceptible stray dogs & 0.25174 & Data \\
\hline$\Psi_{m s}$ & Average number of Maasai dogs that migrate to stray dogs population & 35 & Fitting \\
\hline$\Psi_{s d}$ & $\begin{array}{l}\text { Average number of stray dogs that migrate to domestic dogs } \\
\text { population }\end{array}$ & 17 & Fitting \\
\hline$\Psi_{d s}$ & $\begin{array}{l}\text { Average number of domestic dogs that migrate to stray dogs } \\
\text { population }\end{array}$ & 56 & Fitting \\
\hline$\mu_{c}$ & Average culling rate of stray dogs & 0.01792 & Data \\
\hline
\end{tabular}

We now substitute the parameter values to the expression found in 12 and 13 to have

$$
R_{0}=\frac{1.7864 \times 10^{-4} \times 0.83778234 \times\left(56+35+2.5 \times 10^{2}-17\right)}{0.32 \times(0.22+0.32) \times(0.32+0.83778234)} \approx 1.9
$$

Without any control measure the result of $\mathrm{R}_{0}$ is greater than one which shows that the disease will invade the population. 


$$
R_{e}=\frac{1.7864 \times 10^{-4} \times 0.83778234 \times 0.42\left(56+35+2.5 \times 10^{2}-17\right)}{0.54 \times 1.15778234\left(0.001792+0.0057344+0.0805568+0.032+0.32^{2}\right)} \approx 1.2
$$

With the current vaccination coverage, Re is more than one and this shows that the disease still perseveres. This implies that more efforts should be taken to fight against rabies transmission. We have simulated the effective reproduction number with some variations in vaccination coverage and a combination of vaccination and culling methods. It shows that, by increasing the vaccination of stray dogs, there is a possibility of rabies to die out. The combination of vaccination and culling was found to be the best way to fight against rabies disease transmission in Arusha town. In the simulation, $\mathrm{R}_{0}$ is without any control, $\mathrm{R}_{\mathrm{e} 1}$ is the current $25 \%$ vaccination coverage, $R_{e 2}$ is the $40 \%$ vaccination coverage and $R_{e 3}$ is the combination of $60 \%$ vaccination coverage and $40 \%$ culling.

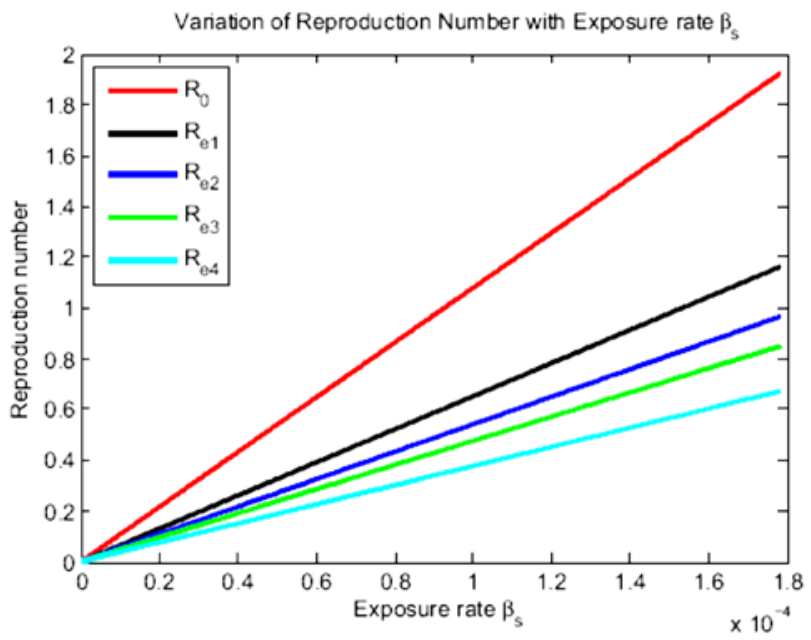

Fig.2. Reproduction number for different vaccination coverages and combination of vaccination and culling.

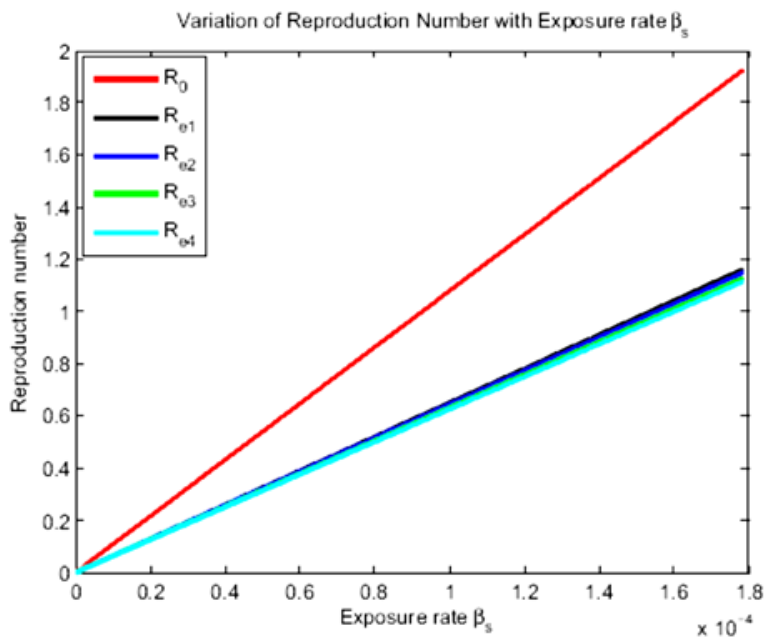

Fig.3. Reproduction number for different culling coverages 
From Figure 2 we can see that $\mathrm{R}_{\mathrm{e} 3}<\mathrm{R}_{\mathrm{e} 2}<\mathrm{R}_{\mathrm{e} 1}<\mathrm{R}_{0}$. This indicates that if we increase vaccination in the stray dog population, the effective reproduction number decreases and become less than one. Due to the high transmission rate of rabies from stray dogs to domestic dogs and Maasai dogs, combination of measures is eagerly recommended as it makes the effective reproduction number less than one. In this case $\mathrm{R}_{0}$ is the current $40 \%$ culling of stray dogs, $\mathrm{R}_{\mathrm{e} 1}$ is the $50 \%$ culling, $\mathrm{R}_{\mathrm{e} 2}$ is the $60 \%$ culling and $\mathrm{R}_{\mathrm{e} 3}$ is the $70 \%$ culling whereby the $25 \%$ current vaccination rate is kept constant.

From Figure 3 we see that culling alone has got a very minute impact in combating rabies transmission risk. The effect observed is for $25 \%$ vaccination coverage only. Therefore, if other practicalities such as costs are disregarded, using a combination of vaccination and culling to control rabies transmission has the highest impact, with increased vaccination coverage.

\section{Stability Analysis}

\subsection{Local Stability of the Disease Free Equilibrium Points}

\section{Theorem 2}

If $\mathrm{R}_{\mathrm{e}}<1$, then

- $\quad$ The disease-free equilibrium $\varepsilon_{0}$ of system 1 is locally asymptotically stable;

- $\quad$ The disease-free equilibrium $\varepsilon_{0}$ of system 1 is globally asymptotically stable in the region $\Phi$

Next we derive the Jacobian matrix of system 1 by differentiating each of the equation of system 1 in terms of state variables $S_{d}, E_{d}, I_{d}, V_{d}, S_{S}, E_{S}, I_{S}, V_{S}, S_{m}, E_{m}, I_{m}, V_{m}$ at the disease free equilibrium point from 6 to have

$$
J_{\varepsilon_{0}}=\left[\begin{array}{cccccccccccc}
-\left(\mu_{d}+\sigma_{d}\right) & 0 & 0 & \omega_{d} & 0 & 0 & A & 0 & 0 & 0 & 0 & 0 \\
0 & H & 0 & 0 & 0 & 0 & B & 0 & 0 & 0 & 0 & 0 \\
0 & \rho_{d} & -\left(\mu_{d}+\delta_{d}\right) & 0 & 0 & 0 & 0 & 0 & 0 & 0 & 0 & 0 \\
\sigma_{d} & o & 0 & -\left(\mu_{d}+\omega_{d}\right) & 0 & 0 & 0 & 0 & 0 & 0 & 0 & 0 \\
0 & 0 & 0 & 0 & G & 0 & C & \omega_{s} & 0 & 0 & 0 & 0 \\
0 & 0 & 0 & 0 & 0 & -\left(\mu_{s}+\rho_{s}\right) & D & 0 & 0 & 0 & 0 & 0 \\
0 & 0 & 0 & 0 & 0 & \rho_{s} & -\left(\mu_{s}+\delta_{s}\right) & 0 & 0 & 0 & 0 & 0 \\
0 & 0 & 0 & 0 & \sigma_{s} & 0 & 0 & I & 0 & 0 & 0 & 0 \\
0 & 0 & 0 & 0 & 0 & 0 & E & 0 & -\left(\mu_{m}+\sigma_{m}\right) & 0 & 0 & \omega_{m} \\
0 & 0 & 0 & 0 & 0 & 0 & F & 0 & 0 & J & 0 & 0 \\
0 & 0 & 0 & 0 & 0 & 0 & 0 & 0 & 0 & \rho_{m} & -\left(\mu_{m}+\delta_{m}\right) & 0 \\
0 & 0 & 0 & 0 & 0 & 0 & 0 & 0 & \sigma_{m} & 0 & 0 & K
\end{array}\right](16)
$$

where 


$$
\begin{aligned}
& A=\frac{-\beta_{d}\left(\alpha_{d}-\Psi_{d s}+\Psi_{m d}+\Psi_{s d}\right)}{\mu_{d}+\sigma_{d}}, B=\frac{\beta_{d}\left(\alpha_{d}-\Psi_{d s}+\Psi_{m d}+\Psi_{s d}\right)}{\mu_{d}+\sigma_{d}}, \\
& C=\frac{-\beta_{s}\left(\mu_{s}+\omega_{s}\right)\left(\Psi_{d s}-\Psi_{s d}+\Psi_{m s}+\alpha_{s}\right)}{\mu_{c}\left(\mu_{s}+\omega_{s}\right)+\mu_{s}\left(\mu_{s}+\sigma_{s}+\omega_{s}\right)}, D=\frac{\beta_{s}\left(\mu_{s}+\omega_{s}\right)\left(\Psi_{d s}-\Psi_{s d}+\Psi_{m s}+\alpha_{s}\right)}{\mu_{c}\left(\mu_{s}+\omega_{s}\right)+\mu_{s}\left(\mu_{s}+\sigma_{s}+\omega_{s}\right)} \\
& E=\frac{-\beta_{s}\left(\alpha_{m}-\Psi_{m d}+\Psi_{m s}\right)}{\mu_{m}+\sigma_{m}}, F=\frac{\beta_{s}\left(\alpha_{m}-\Psi_{m d}+\Psi_{m s}\right)}{\mu_{m}+\sigma_{m}}, G=-\left(\mu_{s}+\rho_{s}\right), \\
& \mathrm{H}=-\left(\mu_{d}+\rho_{d}\right), I=-\left(\mu_{s}+\omega_{s}\right), J=-\left(\mu_{m}+\rho_{m}\right), \mathrm{K}=-\left(\mu_{m}+\omega_{m}\right)
\end{aligned}
$$

The Eigen values of the Jacobian Matrix are:

$$
\left[\begin{array}{c}
-\delta_{d}-\mu_{d} \\
-\mu_{d} \\
-\delta_{m}-\mu_{m} \\
-\mu_{m} \\
-\mu_{d}-\rho_{d} \\
-\mu_{m}-\rho_{m} \\
\frac{1}{2}\left(-\sqrt{4 D \rho_{s}+\left(\delta_{s}-\rho_{s}\right)^{2}}-\delta_{s}-2 \mu_{s}-\rho_{s}\right) \\
\frac{1}{2}\left(\sqrt{4 D \rho_{s}+\left(\delta_{s}-\rho_{s}\right)^{2}}-\delta_{s}-2 \mu_{s}-\rho_{s}\right) \\
-\mu_{d}-\sigma_{d}-\omega_{d} \\
-\mu_{m}-\sigma_{m}-\omega_{m} \\
\frac{1}{2}\left(-\mu_{c}-\sqrt{2 \omega_{s}+\left(\sigma_{s}-\mu_{c}\right)+\left(\sigma_{s}+\mu_{c}\right)^{2}+\omega_{s}^{2}}-2 \mu_{s}-\sigma_{s}-\omega_{s}\right) \\
\frac{1}{2}\left(-\mu_{c}+\sqrt{2 \omega_{s}+\left(\sigma_{s}-\mu_{c}\right)+\left(\sigma_{s}+\mu_{c}\right)^{2}+\omega_{s}^{2}}-2 \mu_{s}-\sigma_{s}-\omega_{s}\right)
\end{array}\right]
$$

From the above Eigen values we see that they are all negative but if

$$
\sqrt{4 D \rho_{s}+\left(\delta_{s}-\rho_{s}\right)^{2}}<\delta_{s}+2 \mu_{s}+\rho_{s}
$$

and

$$
\sqrt{2 \omega_{s}\left(\sigma_{s}-\mu_{c}\right)+\left(\sigma_{s}+\mu_{c}\right)^{2}+\omega_{s}^{2}}<\mu_{c}+2 \mu_{s}+\sigma_{s}+\omega_{s}
$$

then the Disease Free Equilibrium point is locally asymptotically stable. 


\subsection{Global Stability of Disease Free Equilibrium Points}

In this case we employ the method suggested by [19] to scrutinize the global stability of disease free equilibrium point of system 1.Our model represented in system 1 has the following structure.

$$
\left\{\begin{array}{l}
\frac{d x}{d t}=A\left(x-x_{\varepsilon_{0}}\right)+A_{1} y \\
\frac{d y}{d t}=A_{2} y
\end{array}\right.
$$

Where; $x \in \mathbb{R}_{+}$standsforsusceptible and vaccinated individuals. $y \in \mathbb{R}_{+}^{n}$ stands for exposed and infectious individuals. $x_{\varepsilon_{0}}$ is a vector at DFE point $\varepsilon_{0}$ of the vector length $x$. With reference to the system 1 we define $x=\left[\begin{array}{c}S_{d} \\ V_{d} \\ S_{s} \\ V_{s} \\ S_{m} \\ V_{m}\end{array}\right], y=\left[\begin{array}{c}E_{d} \\ I_{d} \\ E_{s} \\ I_{s} \\ V_{m} \\ I_{m}\end{array}\right]$ and $x_{\varepsilon_{0}}=\left[\begin{array}{c}\frac{\alpha_{d}-\Psi_{d s}+\Psi_{m d}+\Psi_{s d}}{\mu_{d}+\sigma_{d}} \\ 0 \\ \frac{\left(\mu_{s}+\omega_{s}\right)\left(\Psi_{d s}-\Psi_{s d}+\Psi_{m s}+\alpha_{s}\right)}{\mu_{c}\left(\mu_{s}+\omega_{s}\right)+\mu_{s}\left(\mu_{s}+\sigma_{s}+\omega_{s}\right)} \\ \frac{\alpha_{s}\left(\Psi_{d s}-\Psi_{s d}+\Psi_{m s}+\alpha_{s}\right)}{\mu_{c}\left(\mu_{s}+\omega_{s}\right)+\mu_{s}\left(\mu_{s}+\sigma_{s}+\omega_{s}\right)} \\ \frac{\alpha_{m}-\Psi_{m d}-\Psi_{m s}}{\mu_{m}+\sigma_{m}} \\ 0\end{array}\right] \rightarrow x-x_{\varepsilon_{0}}=\left[\begin{array}{c}S_{d}-\frac{\alpha_{d}-\Psi_{d s}+\Psi_{m d}+\Psi_{s d}}{\mu_{d}+\sigma_{d}} \\ V_{d} \\ S_{s}-\frac{\left(\mu_{s}+\omega_{s}\right)\left(\Psi_{d s}-\Psi_{s d}+\Psi_{m s}+\alpha_{s}\right)}{\mu_{c}\left(\mu_{s}+\omega_{s}\right)+\mu_{s}\left(\mu_{s}+\sigma_{s}+\omega_{s}\right)} \\ \alpha_{s}\left(\Psi_{d s}-\Psi_{s d}+\Psi_{m s}+\alpha_{s}\right) \\ V_{s}-\frac{1}{\mu_{c}\left(\mu_{s}+\omega_{s}\right)+\mu_{s}\left(\mu_{s}+\sigma_{s}+\omega_{s}\right)} \\ S_{m}-\frac{\alpha_{m}-\Psi_{m d}-\Psi_{m s}}{\mu_{m}+\sigma_{m}} \\ V_{m}\end{array}\right](22)$

To test for global stability of the disease free equilibrium we need to prove the following;

- $A$ should be a matrix with real negative Eigen values,

- $\quad A_{2}$ should be a Metzler matrix.

Using system 1 together with the representation in 21 the two equations can be written as shown below. 


$$
\begin{aligned}
& \left\{\left[\begin{array}{c}
\alpha_{d}+\omega_{d} V_{d}+\Psi_{s d}+\Psi_{m d}-\mu_{d} S_{d}-\sigma_{d} S_{d}-\Psi_{d s}-\beta_{d} S_{d} I_{s} \\
\sigma_{d} S_{d}-\omega_{d} V_{d}-\mu_{d} V_{d} \\
\alpha_{s}+\omega_{s} V_{s}+\Psi_{d s}+\Psi_{m s}-\sigma_{s} S_{s}-\left(\mu_{s}+\mu_{c}\right) S_{s}-\Psi_{s d}-\beta_{s} S_{s} I_{s} \\
\sigma_{s} S_{s}-\omega_{s} V_{s}-\mu_{s} V_{s} \\
\alpha_{m}+\omega_{m} V_{m}-\mu_{m} S_{m}-\Psi_{m s}-\Psi_{m d}-\sigma_{m} S_{m}-\beta_{m} S_{m} I_{s} \\
\sigma_{m} S_{m}-\omega_{m} V_{m}-\mu_{m} V_{m}
\end{array}\right]=A\left[\begin{array}{c}
S_{d}-\frac{\alpha_{d}-\Psi_{d s}+\Psi_{m d}+\Psi_{s d}}{\mu_{d}+\sigma_{d}} \\
V_{d} \\
S_{s}-\frac{\left(\mu_{s}+\omega_{s}\right)\left(\Psi_{d s}-\Psi_{s d}+\Psi_{m s}+\alpha_{s}\right)}{\mu_{c}\left(\mu_{s}+\omega_{s}\right)+\mu_{s}\left(\mu_{s}+\sigma_{s}+\omega_{s}\right)} \\
\alpha_{s}\left(\Psi_{d s}-\Psi_{s d}+\Psi_{m s}+\alpha_{s}\right) \\
V_{s}-\frac{\left.\mu_{s}+\omega_{s}\right)+\mu_{s}\left(\mu_{s}+\sigma_{s}+\omega_{s}\right)}{\mu_{c}\left(\mu_{s}\right)} \\
S_{m}-\frac{\alpha_{m}-\Psi_{m d}-\Psi_{m s}}{\mu_{m}+\sigma_{m}} \\
V_{m}
\end{array}\right]+A_{1}\left[\begin{array}{c}
E_{d} \\
I_{d} \\
E_{s} \\
I_{s} \\
V_{m} \\
I_{m}
\end{array}\right]\right. \\
& {\left[\begin{array}{c}
\beta_{d} S_{d} I_{s}-\left(\mu_{d}+\rho_{d}\right) E_{d} \\
\rho_{a} E_{d}-\left(\mu_{d}+\delta_{d}\right) I_{d} \\
\beta_{s} S_{s} I_{s}-\left(\mu_{s}+\rho_{s}\right) E_{s} \\
\rho_{s} E_{s}-\left(\mu_{s}+\delta_{s}\right) I_{s} \\
\beta_{m} S_{m} I_{s}-\left(\mu_{m}+\rho_{m}\right) E_{m} \\
\rho_{m} E_{m}-\left(\mu_{m}+\delta_{m}\right) I_{m}
\end{array}\right]=A_{2}\left[\begin{array}{c}
E_{d} \\
I_{d} \\
E_{s} \\
I_{s} \\
V_{m} \\
I_{m}
\end{array}\right]}
\end{aligned}
$$

Matrices $A, A_{1}$ and $A_{2}$ are of order $6 \times 6$. Using elements of $x$ of the Jacobian matrix of system 1 at $\varepsilon_{0}$ and representation in 21 we get

$$
\begin{aligned}
& A=\left[\begin{array}{cccccc}
-\left(\mu_{d}+\sigma_{d}\right) & \omega_{d} & 0 & 0 & 0 & 0 \\
\sigma_{d} & -\left(\omega_{d}+\sigma_{d}\right) & 0 & 0 & 0 & 0 \\
0 & 0 & -\left(\mu_{s}+\mu_{c}\right) & \omega_{s} & 0 & 0 \\
0 & 0 & \sigma_{s} & -\left(\omega_{s}+\mu_{s}\right) & 0 & 0 \\
0 & 0 & 0 & 0 & -\left(\mu_{m}+\sigma_{m}\right) & \omega_{m} \\
0 & 0 & 0 & 0 & \sigma_{m} & -\left(\omega_{m}+\mu_{m}\right)
\end{array}\right], A_{1}=\left[\begin{array}{cccccc}
0 & 0 & \beta_{d} S_{d} & 0 & 0 & 0 \\
0 & 0 & 0 & 0 & 0 & 0 \\
0 & 0 & \beta_{s} S_{s} & 0 & 0 & 0 \\
0 & 0 & 0 & 0 & 0 & 0 \\
0 & 0 & \beta_{m} S_{m} & 0 & 0 & 0 \\
0 & 0 & 0 & 0 & 0 & 0
\end{array}\right] \\
& A_{2}=\left[\begin{array}{cccccc}
-\left(\mu_{d}+\rho_{d}\right) & 0 & 0 & \beta_{d} S_{d} & 0 & 0 \\
\rho_{d} & -\left(\mu_{d}+\sigma_{d}\right) & 0 & 0 & 0 & 0 \\
0 & 0 & -\left(\mu_{s}+\rho_{s}\right) & \beta_{s} S_{s} & 0 & 0 \\
0 & 0 & \rho_{s} & -\left(\mu_{s}+\delta_{s}\right) & 0 & 0 \\
0 & 0 & 0 & \beta_{m} S_{m} & -\left(\mu_{m}+\rho_{m}\right) & 0 \\
0 & 0 & 0 & 0 & 0 & -\left(\omega_{m}+\delta_{m}\right)
\end{array}\right]
\end{aligned}
$$

Now we have deduced that, matrix $A$ is an upper triangular matrix with Eigen values being real and negative located in its main diagonal. The Eigen values are $-\left(\mu_{d}+\rho_{d}\right),-\left(\mu_{d}+\sigma_{d}\right),-\left(\mu_{s}+\rho_{s}\right),-\left(\mu_{s}+\delta_{s}\right),-\left(\mu_{m}+\right.$ $\left.\rho_{m}\right)$ and $-\left(\mu_{m}+\delta_{m}\right)$. The off diagonal elements of matrix $A_{2}$ are non-negative since all parameters are positive which proves that it is a Metzler matrix. This also shows that the disease free equilibrium points of system 1 is globally asymptotically stable in the region $\Phi$. This brings us to the following crucial theorem.

\section{Theorem 3}

The disease free equilibrium point is globally asymptotically stable in the region $\Phi$ if $\mathrm{R}_{\mathrm{e}}<1$ and unstable in the region $\Phi$ if $\mathrm{R}_{\mathrm{e}}>1$. 


\section{Endemic Equilibrium Points}

\subsection{Existence of Endemic Equilibrium Points}

We equate the right hand side of system 1 to zero to be able to compute the equilibrium points of system 1 . If the endemic equilibrium points of system 1 exist, they are given by

$\varepsilon_{0}^{*}=\left(S_{d}^{*}, E_{d}^{*}, I_{d}^{*}, V_{d}^{*}, S_{s}^{*}, E_{s}^{*}, I_{s}^{*}, V_{s}^{*}, S_{m}^{*}, E_{m}^{*}, I_{m}^{*}, V_{m}^{*}\right)$

where

$$
\begin{aligned}
& S_{d}^{*}=\frac{\alpha_{d}+\omega_{d} V_{d}^{*}-\Psi_{d s}+\Psi_{m d}+\Psi_{s d}}{\mu_{d}+\beta_{d} I_{s}^{*}+\sigma_{d}}, E_{d}^{*}=\frac{\beta_{d} I_{s}^{*} S_{d}^{*}}{\mu_{d}+\rho_{d}}, I_{d}^{*}=\frac{\rho_{d} E_{d}^{*}}{\delta_{d}+\mu_{d}}, V_{d}^{*}=\frac{\sigma_{d} S_{d}^{*}}{\mu_{d}+\omega_{d}} \\
& S_{s}^{*}=\frac{\alpha_{s}+\omega_{s} V_{s}^{*}-\Psi_{s d}+\Psi_{m s}+\Psi_{d s}}{\mu_{s}+\mu_{c}+\beta_{s} I_{s}^{*}+\sigma_{s}}, E_{s}^{*}=\frac{\beta_{s} I_{s}^{*} S_{s}^{*}}{\mu_{s}+\rho_{s}}, I_{s}^{*}=\frac{\rho_{s} E_{s}^{*}}{\delta_{s}+\mu_{s}}, V_{s}^{*}=\frac{\sigma_{s} S_{s}^{*}}{\mu_{s}+\omega_{s}} \\
& S_{m}^{*}=\frac{\alpha_{m}+\omega_{m} V_{m}^{*}-\Psi_{m s}+\Psi_{m d}}{\mu_{m}+\beta_{m} I_{s}^{*}+\sigma_{m}}, E_{m}^{*}=\frac{\beta_{m} I_{s}^{*} S_{m}^{*}}{\mu_{m}+\rho_{m}}, I_{m}^{*}=\frac{\rho_{m} E_{m}^{*}}{\delta_{m}+\mu_{m}}, V_{m}^{*}=\frac{\sigma_{m} S_{m}^{*}}{\mu_{m}+\omega_{m}}
\end{aligned}
$$

Local Stability of the Endemic Equilibrium

We employed the following theorem as explained by Paul et al., (2016) [20] to describe and prove the local stability of the endemic equilibrium points of system 1.

\section{Theorem 4(Routh-Hurwitz Criterion)}

Given a polynomial $P(\lambda)=\lambda^{n}+a_{1} \lambda^{n-1}+\cdots+a_{n-1} \lambda+a_{n}$

Where the coefficients $a_{i}$ are real constants, $i=1, \ldots, n$ define the $n$ Hurwitz matrices using the coefficients $a_{i}$ of the characteristic polynomial:

$$
H_{1}=\left[a_{1}\right], H_{2}=\left[\begin{array}{ll}
a_{1} & 1 \\
a_{3} & a_{2}
\end{array}\right], H_{3}=\left[\begin{array}{ccc}
a_{1} & 1 & 0 \\
a_{3} & a_{2} & a_{1} \\
a_{5} & a_{4} & a_{3}
\end{array}\right], \ldots, H_{n}=\left[\begin{array}{cccccc}
a_{1} & 1 & 0 & 0 & \cdots & 0 \\
a_{3} & a_{2} & a_{1} & 1 & \cdots & 0 \\
a_{5} & a_{4} & a_{3} & a_{2} & \cdots & 0 \\
\vdots & \vdots & \vdots & \vdots & \ddots & \vdots \\
0 & 0 & 0 & 0 & \cdots & a_{n}
\end{array}\right]
$$

Note that, $a_{i=0} i f j>0$. All of the roots of the polynomial $P(\lambda)$ are negative or have negative real part if the determinants of all Hurwitz matrices are positive: det $\mathrm{H}_{\mathrm{j}}>0 ; \mathrm{j}=0,1,2, \ldots, \mathrm{n}$. More details on Routh-Hurwitz criterion are given by Paul et al., (2016) and Sambo et al., (2013) [21,22]. Consider the first part of system 1. The Jacobian matrix of that part is given by

$$
J \backslash \varepsilon_{0}=\left[\begin{array}{cccc}
-\left(\mu_{d}+\sigma_{d}+\beta_{d} I_{s}\right) & 0 & 0 & \omega_{d} \\
\beta_{d} I_{s} & -\left(\mu_{d}+\rho_{d}\right) & 0 & 0 \\
0 & \rho_{d} & -\left(\mu_{d}+\delta_{d}\right) & 0 \\
0 & 0 & 0 & -\left(\mu_{d}+\omega_{d}\right)
\end{array}\right]
$$


Through computations, we derive the following characteristic polynomial.

$$
\begin{aligned}
& P(\lambda)=\lambda^{4}+A \lambda^{3}+B \lambda^{2}+C \lambda+D \\
& A=\delta_{d}+4 \mu_{d}+\rho_{d}+\beta_{d} I_{s}+\sigma_{d}+\omega_{d} \\
& B= 3 \delta_{d} \mu_{d}+\delta_{d} \rho_{d}+\delta_{d} \sigma_{d}+\delta_{d} \omega_{d}+3 \rho_{d} \mu_{d}+3 \mu_{d} \sigma_{d}+3 \mu_{d} \omega_{d}+6 \mu_{d}^{2}+\rho_{d} \sigma_{d}+\rho_{d} \omega_{d}+I_{s} \beta_{d} \delta_{d}+3 I_{s} \beta_{d} \mu_{d}+I_{s} \beta_{d} \rho_{d} \\
&+I_{s} \beta_{d} \omega_{d}+\sigma_{d} \omega_{d} \\
& C= 2 \delta_{d} \mu_{d} \rho_{d}+2 \delta_{d} \mu_{d} \sigma_{d}+2 \delta_{d} \mu_{d} \omega_{d}+3 \delta_{d} \mu_{d}^{2}+\delta_{d} \rho_{d} \sigma_{d}+\delta_{d} \sigma_{d} \omega_{d}+\delta_{d} \rho_{d} \omega_{d}+2 \mu_{d} \rho_{d} \sigma_{d}+2 \mu_{d} \rho_{d} \omega_{d}+3 \mu_{d}^{2} \rho_{d}+ \\
& 2 \mu_{d} \sigma_{d} \omega_{d}+3 \mu_{d}^{2} \sigma_{d}+3 \mu_{d}^{2} \omega_{d}+4 \mu_{d}^{3}+\rho_{d} \sigma_{d} \omega_{d}+2 I_{s} \beta_{d} \delta_{d} \mu_{d}+I_{s} \beta_{d} \delta_{d} \rho_{d}+I_{s} \beta_{d} \delta_{d} \mu_{d} \omega_{d}+2 I_{s} \beta_{d} \mu_{d} \rho_{d}+ \\
& 2 I_{s} \beta_{d} \mu_{d} \omega_{d}+3 I_{s} \beta_{d} \mu_{d}^{2}+I_{s} \beta_{d} \rho_{d} \mu_{d} \\
& D= \delta_{d} \mu_{d} \rho_{d} \sigma_{d}+\delta_{d} \mu_{d} \rho_{d} \omega_{d}+\delta_{d} \mu_{d}^{2} \rho_{d}+\delta_{d} \mu_{d} \sigma_{d} \omega_{d}+\delta_{d} \mu_{d}^{2} \sigma_{d}+\delta_{d} \mu_{d}^{2} \omega_{d}+\delta_{d} \mu_{d}^{3}+\delta_{d} \rho_{d} \sigma_{d} \omega_{d}+\mu_{d} \rho_{d} \sigma_{d} \omega_{d}+ \\
& \mu_{d}^{2} \rho_{d} \sigma_{d}+\mu_{d}^{2} \rho_{d} \omega_{d}+\mu_{d}^{3} \rho_{d}+\mu_{d}^{2} \sigma_{d} \omega_{d}+\mu_{d}^{3} \sigma_{d}+\mu_{d}^{3} \omega_{d}+\mu_{d}^{4}+I_{s} \beta_{d} \delta_{d} \mu_{d} \rho_{d}+I_{s} \beta_{d} \delta_{d} \mu_{d} \omega_{d}+I_{s} \beta_{d} \delta_{d} \mu_{d}^{2}+ \\
& I_{s} \beta_{d} \delta_{d} \rho_{d} \omega_{d}+I_{s} \beta_{d} \mu_{d} \rho_{d} \omega_{d}+I_{s} \beta_{d} \mu_{d}^{2} \omega_{d}+I_{s} \beta_{d} \mu_{d}^{3}
\end{aligned}
$$

From the characteristic polynomial represented in 31 we have the following Hurwitz matrix

$$
H_{4}=\left[\begin{array}{llll}
A & 1 & 0 & 0 \\
C & B & A & 1 \\
0 & D & C & B \\
0 & 0 & 0 & D
\end{array}\right]
$$

The determinant of the Hurwitz matrix is $D\left(\mathrm{ABC}-\mathrm{C}^{2}-\mathrm{A}^{2} \mathrm{D}\right)$.

From the Routh-Hurwitz criteria of Theorem 4, we see that the determinant of Hurwitz matrix will be positive if the following conditions hold true. $A>0, C>0, D>0$ and $A B C>C^{2}+A^{2} D$. Recall that all parameters of our model and all coefficients of the characteristic polynomial are positive as shown in equation 32. We combine all requirements and deduce that all roots of the polynomial represented in 31 are negative and hence we prove that the first part of system 1 is locally asymptotically stable. Moreover, we consider the second part of system 1 . The Jacobian matrix is given by

$$
J \backslash \varepsilon_{0}=\left[\begin{array}{cccc}
-\left(\sigma_{s}+\mu_{s}+\mu_{c}+\beta_{s} I_{s}\right) & 0 & \beta_{s} S_{s} & \omega_{s} \\
\beta_{s} I_{s} & -\left(\mu_{s}+\rho_{s}\right) & -\beta_{s} S_{s} & 0 \\
0 & \rho_{s} & -\left(\mu_{s}+\delta_{s}\right) & 0 \\
\sigma_{s} & 0 & 0 & -\left(\mu_{s}+\omega_{s}\right)
\end{array}\right]
$$

Consider the characteristic polynomial

$$
P(\lambda)=\lambda^{4}+A_{1} \lambda^{3}+B_{1} \lambda^{2}+C_{1} \lambda+D_{1}
$$




$$
\begin{aligned}
& A_{1}=\mu_{c}+\beta_{s} I_{s}+\delta_{s}+4 \mu_{s}+\rho_{s}+\sigma_{s}+\omega_{s} \\
& B_{1}=\mu_{c} \delta_{s}+\mu_{c} \rho_{s}+\mu_{c} \omega_{s}+3 \mu_{c} \mu_{s}+\beta_{s} I_{s} \delta_{s}+3 \beta_{s} I_{s} \mu_{s}+\beta_{s} I_{s} \rho_{s}+3 \delta_{s} \mu_{s}+\delta_{s} \rho_{s}+\delta_{s} \sigma_{s}+\delta_{s} \omega_{s}+3 \mu_{s} \rho_{s} \\
& +3 \mu_{s} \sigma_{s}+3 \mu_{s} \omega_{s}+6 \mu_{s}^{2}+\rho_{s} \sigma_{s}+\rho_{s} \omega_{s}-\beta_{s} \rho_{s} S_{s} \\
& C_{1}=\mu_{c} \delta_{s} \rho_{s}+\mu_{c} \delta_{s} \omega_{s}+2 \mu_{c} \delta_{s} \mu_{s}+\mu_{c} \rho_{s} \omega_{s}+2 \mu_{c} \mu_{s} \rho_{s}+2 \mu_{c} \mu_{s} \omega_{s}+3 \mu_{c} \mu_{s}^{2}-\mu_{c} \beta_{s} \rho_{s} S_{s}+2 \beta_{s} I_{s} \delta_{s} \mu_{s}+ \\
& +\beta_{s} I_{s} \delta_{s} \rho_{s}+\beta_{s} I_{s} \delta_{s} \omega_{s}+2 \beta_{s} I_{s} \mu_{s} \rho_{s}+2 \beta_{s} I_{s} \mu_{s} \omega_{s}+3 \beta_{s} I_{s} \mu_{s}^{2}+\beta_{s} I_{s} \rho_{s} \omega_{s}+2 \delta_{s} \mu_{s} \rho_{s}+2 \delta_{s} \mu_{s} \sigma_{s}+ \\
& 2 \delta_{s} \mu_{s} \omega_{s}+3 \delta_{s} \mu_{s}^{2}+\delta_{s} \rho_{s} \sigma_{s}+\delta_{s} \rho_{s} \omega_{s}+2 \mu_{s} \rho_{s} \sigma_{s}+2 \mu_{s} \rho_{s} \omega_{s}+3 \mu_{s}^{2} \rho_{s}+3 \mu_{s}^{2} \sigma_{s}+3 \mu_{s}^{2} \omega_{s}+4 \mu_{s}^{3}- \\
& 2 \beta_{s} \mu_{s} \rho_{s} S_{s}-\beta_{s} \rho_{s} \sigma_{s} S_{s}-\beta_{s} \rho_{s} S_{s} \omega_{s} \\
& D_{1}=\mu_{c} \delta_{s} \rho_{s} \omega_{s}+\mu_{c} \delta_{s} \mu_{s} \rho_{s}+\mu_{c} \delta_{s} \mu_{s} \omega_{s}+\mu_{c} \delta_{s} \mu_{s}^{2}+\mu_{c} \mu_{s} \rho_{s} \omega_{s}+\mu_{c} \mu_{s}^{2} \rho_{s}+\mu_{c} \mu_{s}^{2} \omega_{s}+\mu_{c} \mu_{s}^{3}-\mu_{c} \beta_{s} \rho_{s} S_{s} \omega_{s} \\
& -\mu_{c} \beta_{s} \mu_{s} \rho_{s} S_{s}+\beta_{s} I_{s} \delta_{s} \mu_{s} \rho_{s}+\beta_{s} I_{s} \delta_{s} \mu_{s} \omega_{s}+\beta_{s} I_{s} \delta_{s} \mu_{s}^{2}+\beta_{s} I_{s} \delta_{s} \rho_{s} \omega_{s}+\beta_{s} I_{s} \mu_{s} \rho_{s} \omega_{s}+\beta_{s} I_{s} \mu_{s}^{2} \rho_{s}+ \\
& \beta_{s} I_{s} \mu_{s}^{2} \omega_{s}+\beta_{s} I_{s} \mu_{s}^{3}+\delta_{s} \mu_{s} \rho_{s} \sigma_{s}+\delta_{s} \mu_{s} \rho_{s} \omega_{s}+\delta_{s} \mu_{s}^{2} \rho_{s}+\delta_{s} \mu_{s}^{2} \sigma_{s}+\delta_{s} \mu_{s}^{2} \omega_{s}+\delta_{s} \mu_{s}^{3}+\mu_{s}^{2} \rho_{s} \sigma_{s}+\mu_{s}^{2} \rho_{s} \omega_{s} \\
& +\mu_{s}^{3} \rho_{s}+\mu_{s}^{3} \sigma_{s}+\mu_{s}^{3} \omega_{s}+\mu_{s}^{4}-\beta_{s} \mu_{s} \rho_{s} \sigma_{s} S_{s}-\beta_{s} \mu_{s} \rho_{s} S_{s} \omega_{s}-\beta_{s} \mu_{s}^{2} \rho_{s} S_{s}
\end{aligned}
$$

From the characteristic polynomial represented by 35 we have the Hurwitz matrix being given by

$$
H_{5}=\left[\begin{array}{cccc}
A_{1} & 1 & 0 & 0 \\
C_{1} & B_{1} & A_{1} & 1 \\
0 & D_{1} & C_{1} & B_{1} \\
0 & 0 & 0 & D_{1}
\end{array}\right]
$$

The determinant of the Hurwitz matrix is given by $D_{1}\left(A_{1} B_{1} C_{1}-C_{1}^{2}-A_{1}^{2} D_{1}\right)$. With reference to Theorem 4 the determinant of Hurwitz matrix become positive iff $A_{1}>0, C_{1}>0, D_{1}>0$ and $A_{1} B_{1} C_{1}>C_{1}^{2}+A_{1}^{2} D_{1}$. Again, since $A_{1}>0$,

$$
\begin{aligned}
& B_{1}>0 \text { iff } \mu_{c} \delta_{s}+\mu_{c} \rho_{s}+\mu_{c} \omega_{s}+3 \mu_{c} \mu_{s}+I_{s} \beta_{s} \delta_{s}+3 I_{s} \beta_{s} \mu_{s}+I_{s} \beta_{s} \rho_{s}+I_{s} \beta_{s} \omega_{s}+3 \delta_{s} \mu_{s}+\delta_{s} \rho_{s}+\delta_{s} \sigma_{s}+ \\
& \delta_{s} \omega_{s}+3 \mu_{s} \rho_{s}+3 \mu_{s} \sigma_{s}+3 \mu_{s} \omega_{s}+6 \mu_{s}^{2}+\rho_{s} \sigma_{s}+\rho_{s} \omega_{s}>\beta_{s} \rho_{s} S_{s} \\
& C_{1}>0 \text { iff } \mu_{c} \delta_{s} \rho_{s}+\mu_{c} \delta_{s} \omega_{s}+2 \mu_{c} \delta_{s} \mu_{s}+\mu_{c} \rho_{s} \omega_{s}+2 \mu_{c} \mu_{s} \rho_{s}+2 \mu_{c} \mu_{s} \omega_{s}+3 \mu_{c} \mu_{s}^{2}+2 I_{s} \beta_{s} \delta_{s} \mu_{s}+I_{s} \beta_{s} \delta_{s} \rho_{s} \\
& +I_{s} \beta_{s} \delta_{s} \omega_{s}+2 I_{s} \beta_{s} \mu_{s} \rho_{s}+2 I_{s} \beta_{s} \mu_{s} \omega_{s}+3 I_{s} \beta_{s} \mu_{s}^{2}+I_{s} \beta_{s} \rho_{s} \omega_{s}+2 \delta_{s} \mu_{s} \rho_{s}+2 \delta_{s} \mu_{s} \sigma_{s}+2 \delta_{s} \mu_{s} \omega_{s}+ \\
& 3 \delta_{s} \mu_{s}^{2}+\delta_{s} \rho_{s} \sigma_{s}+\delta_{s} \rho_{s} \omega_{s}+2 \mu_{s} \rho_{s} \sigma_{s}+2 \mu_{s} \rho_{s} \omega_{s}+3 \mu_{s}^{2} \rho_{s}+3 \mu_{s}^{2} \sigma_{s}+3 \mu_{s}^{2} \omega_{s}+4 \mu_{s}^{3}>2 \beta_{s} \mu_{s} \rho_{s} S_{s}+ \\
& \beta_{s} \rho_{s} \sigma_{s} S_{s}+\beta_{s} \rho_{s} S_{s} \omega_{s}+\mu_{c} \beta_{s} \rho_{s} S_{s} \\
& D_{1}>0 \text { iff } \mu_{c} \delta_{s} \rho_{s} \omega_{s}+\mu_{c} \delta_{s} \mu_{s} \rho_{s}+\mu_{c} \delta_{s} \mu_{s} \omega_{s}+\mu_{c} \delta_{s} \mu_{s}^{2}+\mu_{c} \mu_{s} \rho_{s} \omega_{s}+\mu_{c} \mu_{s}^{2} \rho_{s}+\mu_{c} \mu_{s}^{2} \omega_{s}+\mu_{c} \mu_{s}^{3}+I_{s} \beta_{s} \delta_{s} \mu_{s} \rho_{s} \\
& +I_{s} \beta_{s} \delta_{s} \mu_{s} \omega_{s}+I_{s} \beta_{s} \delta_{s} \mu_{s}^{2}+I_{s} \beta_{s} \delta_{s} \rho_{s} \omega_{s}+I_{s} \beta_{s} \mu_{s} \rho_{s} \omega_{s}+I_{s} \beta_{s} \mu_{s}^{2} \rho_{s}+I_{s} \beta_{s} \mu_{s}^{2} \omega_{s}+I_{s} \beta_{s} \mu_{s}^{3}+\delta_{s} \mu_{s} \rho_{s} \sigma_{s} \\
& +\delta_{s} \mu_{s} \rho_{s} \omega_{s}+\delta_{s} \mu_{s}^{2} \sigma_{s}+\delta_{s} \mu_{s}^{2} \omega_{s}+\delta_{s} \mu_{s}^{2} \rho_{s}+\delta_{s} \mu_{s}^{3}+\mu_{s}^{2} \rho_{s} \sigma_{s}+\mu_{s}^{2} \rho_{s} \omega_{s}+\mu_{s}^{3} \rho_{s}+\mu_{s}^{3} \sigma_{s}+\mu_{s}^{3} \omega_{s}+\mu_{s}^{4} \\
& >\beta_{s} \mu_{s} \rho_{s} \sigma_{s} S_{s}+\beta_{s} \mu_{s} \rho_{s} S_{s} \omega_{s}+\beta_{s} \mu_{s}^{2} \rho_{s} S_{s}+\mu_{c} \beta_{s} \rho_{s} S_{s} \omega_{s}+\mu_{c} \beta_{s} \mu_{s} \rho_{s} S_{s}
\end{aligned}
$$

When all conditions hold, similarly $\mathrm{A}_{1} \mathrm{~B}_{1} \mathrm{C}_{1}>\mathrm{C}_{1}^{2}+\mathrm{A}_{1}^{2} \mathrm{D}_{1}$ holds. Hence we can conclude that all roots of polynomial 35 are negative. This verifies that the second part of system 1 is locally asymptotically stable. Using the same procedure for the third part of system 1 will result in the same conclusion.

Therefore, we generally conclude that the endemic equilibrium point of system 1 is locally asymptotically stable. 


\section{Conclusion}

In this paper we have formulated and analyzed a deterministic mathematical model for the dynamics of rabies transmission. The model consists of domestic dogs, stray dogs and Maasai dogs' population. The model intended to show the contribution of vaccination, culling and their combination towards the control of rabies transmission.

The basic reproduction number and the effective reproduction number have been computed using next generation matrix operator. The results depend on the parameter values of stray dogs' population. This is because, epidemiologically, stray dogs are the main source of dog-rabies transmission. This tells us that more effort on controlling dog-rabies transmission should be put into the stray dog population [12].

Using our set of parameter values, the basic reproduction number $\mathrm{R}_{0}$ and the effective reproduction number $\mathrm{R}_{\mathrm{e}}$ were computed and found to be 1.9 and 1.2 respectively. This indicates that the disease is endemic [8].

The numerical simulation for the reproduction number indicates that the combination of at least $60 \%$ vaccination and culling of up to $40 \%$ of the dog population would be the best measure to control dog-rabies transmission in Arusha region. However, this does not take into account feasibility and economic and sociocultural aspects.

We have computed the disease free and endemic equilibrium points. With the use of Routh Hurwitz criteria, the endemic equilibrium points are locally asymptotically stable if these conditions hold.

\section{Acknowledgments}

The authors thank MWECAU for the sponsorship of studies. Also authors appreciate the data provision support from the ministry of agriculture, livestock development and fisheries of the United Republic of Tanzania and Jens Fissenebert of Mbwa wa Africa.

\section{References}

[1] Abta, A., Laarabi, H., and Talibi Alaoui, H. (2014). The hopf bifurcation analysis and optimal control of a delayed sir epidemic model.International Journal of Analysis, 2014.

[2] Addo, K. M. (2012). An SEIR Mathematical Model for dog Rabies; Case Study: Bongo District, Ghana. $\mathrm{PhD}$ thesis.

[3] Allen, L. J. and Van den Driessche, P. (2008). The basic reproduction number in some discrete-time epidemic models. Journal of Difference Equations and Applications, 14(10-11):1127-1147.

[4] Amaku, M., Dias, R. A., and Ferreira, F. (2010). Dynamics and control of stray dog populations. Mathematical Population Studies, 17(2):69-78.

[5] Aweya, J., Ouellette, M., and Montuno, D. Y. (2004). Design and stability analysis of a rate control algorithm using the routh-hurwitz stability criterion. IEEE/ACM transactions on networking, 12(4):719 732.

[6] Cintr'on-Arias, A., Castillo-Ch'avez, C., Bettencourt, L. M., Lloyd, A. L., and Banks, H. (2009). The estimation of the effective reproductive number from disease outbreak data. Math BiosciEng, 6(2):261282.

[7] Cowling, B. J., Lau, M. S., Ho, L.-M., Chuang, S.-K., Tsang, T., Liu, S.-H., Leung, P.-Y., Lo, S.-V., and Lau, E. H. (2010). The effective reproduction number of pandemic influenza: prospective estimation. Epidemiology (Cambridge, Mass.), 21(6):842.

[8] Ega, T. T., Luboobi, L. S., and Kuznetsov, D. (2015). Modeling the dynamics of rabies transmission with vaccination and stability analysis. Applied and Computational Mathematics, 4(6):409-419. 
[9] El-Marhomy, A. A. and Abdel-Sattar, N. E. (2004). Stability analysis of rotor-bearing systems via routhhurwitz criterion.Applied Energy, 77(3):287-308.

[10] Gil, J. J., Avello, A., Rubio, A., and Florez, J. (2004). Stability analysis of a 1 dof haptic interface using the routh-hurwitz criterion. IEEE transactions on control systems technology, 12(4):583-588.

[11] Hampson, K., Dushoff, J., Cleaveland, S., Haydon, D. T., Kaare, M., Packer, C., and Dobson, A. (2009). Transmission dynamics and prospects for the elimination of canine rabies. PLoS biology, 7(3):e1000053.

[12] Hou, Q., Jin, Z., and Ruan, S. (2012). Dynamics of rabies epidemics and the impact of control efforts in guangdong province, china. Journal of theoretical biology, 300:39-47.

[13] Iggidr, A., Mbang, J., Sallet, G., and Tewa, J.-J. (2007). Multi-compartment models. Discrete and Continuous Dynamical Systems-Series S, 2007(Special):506-519.

[14] Knobel, D. L., Cleaveland, S., Coleman, P. G., F`evre, E. M., Meltzer, M. I., Miranda, M. E. G., Shaw, A., Zinsstag, J., and Meslin, F.-X. (2005). Re-evaluating the burden of rabies in Africa and Asia. Bulletin of the World health Organization, 83:360 - 368.

[15] L'echenne, M., Oussiguere, A., Naissengar, K., Mindekem, R., Mosimann, L., Rives, G., Hattendorf, J., Moto, D. D., Alfaroukh, I. O., Zinsstag, J., et al. (2016). Operational performance and analysis of two rabies vaccination campaigns in ndjamena, chad. Vaccine, 34(4):571-577.

[16] Lembo, T., Hampson, K., Kaare, M. T., Ernest, E., Knobel, D., Kazwala, R. R., Haydon, D. T., and Cleaveland, S. (2010). The feasibility of canine rabies elimination in Africa: dispelling doubts with data. PLoS neglected tropical diseases, 4(2):e626.

[17] Leung, T. and Davis, S. A. (2017). Rabies vaccination targets for stray dog populations. Frontiers in veterinary science, $4: 52$.

[18] Morters, M. K., Restif, O., Hampson, K., Cleaveland, S., Wood, J. L., and Conlan, A. J. (2013). Evidencebased control of canine rabies: a critical review of population density reduction. Journal of animal ecology, 82(1):6-14.

[19] Mpolya, E. A., Lembo, T., Lushasi, K., Mancy, R., Mbunda, E. M., Makungu, S., Maziku, M., Sikana, L., Jaswant, G., Townsend, S., et al. (2017). Toward elimination of dog-mediated human rabies: experiences from implementing a large-scale demonstration project in southern Tanzania. Frontiers in veterinary science, 4:21.

[20] Paul, M., Majumder, S. S., Sau, S., Nandi, A. K., and Bhadra, A. (2016). High early life mortality in freeranging dogs is largely influenced by humans. Scientific reports, 6:19641.

[21] Sambo, M., Cleaveland, S., Ferguson, H., Lembo, T., Simon, C., Urassa, H., and Hampson, K. (2013). The burden of rabies in Tanzania and its impact on local communities. PLoS neglected tropical diseases, 7(11):e2510.

[22] Totton, S. C.,Wandeler, A. I., Zinsstag, J., Bauch, C. T., Ribble, C. S., Rosatte, R. C., and McEwen, S. A. (2010). Stray dog population demographics in Jodhpur, India following a population control/rabies vaccination program. Preventive veterinary medicine, 97(1):51-57.

[23] Tulu, A. M. and Koya, P. R. (2017). The impact of infective immigrants on the spread of dog rabies. American Journal of Applied Mathematics, 5(3):68.

[24] Van den Driessche, P. and Watmough, J. (2008).Further notes on the basic reproduction number. In Mathematical epidemiology, pages 159-178. Springer.

[25] Zhang, J., Jin, Z., Sun, G.-Q., Zhou, T., and Ruan, S. (2011). Analysis of rabies in china: transmission dynamics and control. PLoS one, 6(7):e20891.

[26] Zinsstag, J., D"urr, S., Penny, M., Mindekem, R., Roth, F., Gonzalez, S. M., Naissengar, S., and Hattendorf, J. (2009). Transmission dynamics and economics of rabies control in dogs and humans in an African city. Proceedings of the National Academy of Sciences, 106(35):14996-15001.

[27] P. van den Driessche, J. Watmough, A simple sis epidemic model with a backward bifurcation, Journal of Mathematical Biology 40 (2000) 525-540. 


\section{Authors' Profiles}

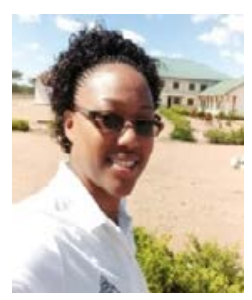

Edwiga Kishinda Renald is a student at Nelson Mandela African Institution of Science and Technology (NM-AIST) pursuing a master degree in Applied Mathematics and Computational Sciences. She received her first degree in Mathematics and Statistics at Mwenge Catholic University where she currently works as a tutorial assistant in Mathematics and Statistics department. Her interested area of research is mathematical modelling of infectious diseases.

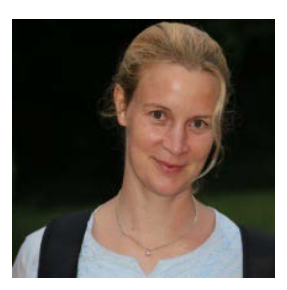

Katharina Kreppel is the postdoctoral training coordinator for the Afrique One-ASPIRE programme at NM-AIST. Dr Kreppel holds a PhD in Epidemiology from the School of Veterinary Sciences, University of Liverpool, UK. Her research interest lie in One Health and understanding the ecology and behavior of tropical disease vectors and relate these to the epidemiology of diseases. She has a total of 10 years' experience of field-based research in Africa on the effects of climate and the environment on vector ecology and disease epidemiology.

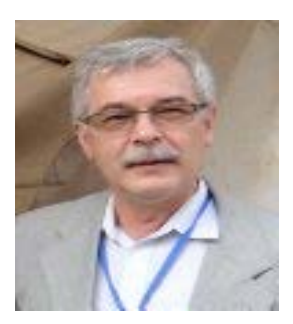

Dmitry Kuznetsov is an associate professor at NM-AIST. Prof. Kuznetsov holds a PhD in Mathematics from the Yaroslavl State Pedagogical University, Russia. His research interests lie in applied and computational mathematics. He has a total of 10 years' experience of teaching mathematics in Rwanda and Tanzania.

How to cite this paper: Edwiga Kishinda Renald, Katharina Kreppel, Dmitry Kuznetsov, " Desirable DogRabies Control Methods in an Urban setting in Africa - a Mathematical Model ", International Journal of Mathematical Sciences and Computing(IJMSC), Vol.6, No.1, pp.49-67, 2020. DOI: 10.5815/ijmsc.2020.01.05 